\title{
Énée face à Didon (Énéide IV, 333-361)
}

\section{Claude Loutsch}

\section{(2) OpenEdition}

Journals

Édition électronique

URL : http://journals.openedition.org/rhetorique/179

ISSN : 2270-6909

Éditeur

UGA Éditions/Université Grenoble Alpes

Édition imprimée

ISBN : 978-2-84310-263-9

Référence électronique

Claude Loutsch, «Énée face à Didon (Énéide IV, 333-361) », Exercices de rhétorique [En ligne], 2 | 2013, mis en ligne le 28 janvier 2014, consulté le 12 septembre 2020. URL : http://journals.openedition.org/ rhetorique/179

Ce document a été généré automatiquement le 12 septembre 2020.

\section{(c) (i) (2)(2)}

Les contenus de la revue Exercices de rhétorique sont mis à disposition selon les termes de la Licence Creative Commons Attribution - Pas d'Utilisation Commerciale - Partage dans les Mêmes Conditions 4.0 International. 


\title{
Énée face à Didon (Énéide IV, 333-361)
}

\author{
Claude Loutsch
}

1 Au livre IV de l'Énéide ${ }^{1}$, Virgile donne au récit de la rupture entre Didon et Énée la forme dramatique. Trois discours s'y succèdent: un premier discours de Didon juste après qu'elle vient d'apprendre par des voies détournées (la « Rumeur », " Fama ») les préparatifs d'Énée pour quitter Carthage et mettre le cap sur l'Italie, discours dans lequel elle clame toute sa colère et le supplie de renoncer à son projet (305-330); la réponse d'Énée qui rejette les reproches de Didon et justifie son départ (333-360); enfin, la riposte de Didon qui crie son désespoir et maudit son ancien amant (365-387).

2 Auparavant, le poète a raconté comment Jupiter avait décidé de mettre un terme au séjour d'Énée à Carthage et avait envoyé Mercure pour en transmettre l'ordre à Énée. Mercure trouva ce dernier, portant de somptueux habits, cadeaux de Didon, en train de surveiller la construction de la ville et des fortifications de Carthage (260). La réaction d'Énée est immédiate et sans hésitation (281-282) : « Ardet abire fuga dulcesque relinquere terras, / attonitus tanto monitu imperioque deorum ", "il brûle de prendre la fuite et de partir, de quitter ces douces terres, foudroyé par l'avertissement et l'ordre si impérieux des dieux ». Sa seule préoccupation et hésitation : comment annoncer son départ à la reine déchaînée (283 : "furentem ») ? comment trouver les mots justes pour l'aborder (284 : "quae prima exordia sumat ", " par où commencer ») ? Il donne à ses hommes l'ordre d'entamer aussitôt les préparatifs pour le départ, mais il retarde encore le moment pour parler à Didon, attendant « quae mollissima fandi tempora, quis rebus dexter modus» (293-294: « les moments les plus propices pour parler, le moyen le plus adroit pour son affaire »). Précaution bien inutile, car la Rumeur irrévérencieuse (298: «impia Fama») se charge de mettre Didon au courant de ce qui se trame à son insu. Celle-ci, hors d'elle (300-301: "saeuit inops animi totamque incensa per urbem / bacchatur ", " elle se déchaîne, perd toute sa raison et erre enflammée, telle une Bacchante, à travers toute la ville »), demande aussitôt des explications à Énée.

3 Le poète décrit ainsi non seulement la situation immédiate dans laquelle Énée va prendre la parole, mais aussi comment le héros a longtemps à l'avance médité son 
discours ${ }^{2}$ : les difficultés qu'il imagine, une évaluation réaliste de la situation (283-284); Énée passe et repasse dans son esprit différentes idées, différents projets de discours (285-286), avant de décider de ne rien faire dans l'immédiat, d'attendre le moment favorable (293-294), le kairós.

\section{Aperçu d'ensemble}

\section{Aperçu des trois discours : Didon, Énée, Didon}

4 Le premier discours de Didon ${ }^{3}$ commence ex abrupto par un exorde (305-308) où elle accuse Énée, dans une première partie ("ab aduersarii persona ", "à partir de la partie adverse »), de dissimulatio, de perfidia et de nefas (de "sacrilège ») et, dans une deuxième partie ( $a$ sua persona», «à partir de soi-même»), d'oublier leur amour, leur engagement et son sort à elle. La partie principale est une tentative de le dissuader de partir, d'abord en le raisonnant (en lui faisant voir les dangers de la navigation en plein hiver, à laquelle il ne songerait pas pour regagner Troie, même si Troie était toujours debout : 309-313), ensuite en le suppliant, en essayant de l'infléchir sur son sort à elle : elle lui rappelle leur passé (leur mariage, ses mérites envers lui : 314-318) et l'implore d'avoir pitié d'elle et de songer à ce qu'elle va devenir, de ne pas l'abandonner à ses ennemis, de songer à son pudor ("honneur») et à sa fama ("réputation») qu'elle a sacrifiés pour lui (318-326). Enfin, elle conclut par un vœu déchirant : si seulement elle avait un enfant de lui, elle ne se sentirait pas complètement abandonnée (327-3304).

5 Ce discours n'est pas un réquisitoire (genre judiciaire), mais une suasoria, une exhortation (genre délibératif) à l'adresse d'Énée pour qu'il renonce à son projet de partir : elle y fait valoir plusieurs bonnes raisons pour rester auprès d'elle.

\section{Le discours d'Énée}

Virgile note la réaction d'Énée à ce discours passionné : ne songeant qu'à la mise en garde de Jupiter, il regarde droit devant lui et fait un grand effort pour refouler son chagrin (332 : «obnixus curam sub corde premebat ${ }^{5}$ ", « à grand effort, il tâchait d'étouffer le tourment au fond de son cœur »). Après un long moment de silence (suggéré par la position de "tandem ", « enfin ", en tête du vers 333), il répond brièvement ${ }^{6}$. Sa réponse relève d'un genre différent de celui du discours de Didon: il défend son projet de départ et énumère les raisons qui font qu'il lui est impossible d'y renoncer. Dans l'exorde, il se montre plein d'égards pour son interlocutrice: il déclare être parfaitement conscient de l'ampleur de ses mérites à elle à son égard et il lui promet de ne jamais l'oublier. Un exorde qui doit apaiser Didon. Le ton est très respectueux, mais en même temps Énée prend ses distances et montre clairement dans quel rôle il s'est désormais glissé : dans un premier temps, il appelle Didon non pas de son nom, mais de son titre (334: « regina »), et ce faisant il la renvoie à son rôle public de reine. Et pour parler de leurs relations, il parle au passé (335: «promeritam », " que tu aies mérité de moi »), sous-entendant par là que leur relation n'a plus d'avenir. Le ton est d'une politesse irréprochable, mais toute familiarité en est exclue : le dux s'adresse à la regina, du moins au présent et à l'avenir; peut-être par le choix du nom Elissa dans un deuxième temps (335), reconnaît-il une certaine intimité pour le passé. 
7 Suit une sorte de propositio au vers 337 : «pro re pauca loquor », «sur le fond, j'ai peu à dire "; sa réponse sera brève. La brièveté est le fait de l'homme sûr de lui-même ; elle implique tout refus de verbiage et elle est gage de sincérité. "Pro re », "sur le fond" ${ }^{7}$ : donc il va se justifier, se défendre contre des accusations contenues dans la suasoria de Didon. Il rejette d'abord l'accusation d'avoir voulu dissimuler comme un voleur sa fuite, ensuite celle de rompre les liens du mariage. À la première accusation, il oppose une simple dénégation («neque abscondere speraui », " je n'ai pas espéré dissimuler $\left.{ }^{8} »\right)$; à la deuxième accusation, il rappelle qu'il n'y a jamais eu de cérémonie de mariage entre eux et qu'il n'est pas venu en Libye dans l'intention de l'épouser. Ici encore un ton sec et déterminé, qui exclut toute longue discussion: c'est qu'il s'agit de défendre sa réputation. On notera la réserve vis-à-vis de Didon : il ne l'accuse pas de se tromper, il redresse simplement ses affirmations et insinuations, mais cela de manière autoritaire (338: « ne finge », « ne t'imagine pas $\left.^{9} »\right)$.

8 Si Énée balaie péremptoirement la prétendue intention de dissimuler sa fuite, le fait de nier avoir jamais eu l'intention de se marier va bien au-delà d'une simple dénégation : il s'agit d'une manière pudique de dire brutalement à Didon qu'elle s'est trompée en assimilant leur liaison à un mariage en bonne et due forme, donc de mettre les points sur les i et de requalifier le prétendu mariage ${ }^{10}$.

9 Au ton ferme de la première partie succède une deuxième partie où la défense est plus circonstanciée: en réplique à la remarque de Didon qu'il courra plus de risques pour gagner l'Italie qu'il n'en accepterait de courir pour regagner Troie, il affirme solennellement et avec beaucoup de nostalgie que si les destins l'autorisaient à mener sa vie à sa guise, il s'occuperait d'abord de Troie et des tombeaux des siens, il aurait déjà reconstruit les demeures de Priam et fait revivre Pergame (340-344). Donc, ici encore, le souci de ne pas apparaître infidèle à ses idéaux, à sa patrie, mais aussi l'affirmation capitale qu'il n'est pas libre de choisir la vie qu'il mène. En d'autres termes qu'il n'est pas responsable de ses choix, que d'autres lui dictent ce qu'il doit faire. Il énumère ces autres dans les vers qui suivent : $1^{\circ}$ l'oracle d'Apollon qui lui a ordonné de gagner l'Italie et lui a fait comprendre que c'est là où doit être son cœur et sa patrie (345-347). Il ouvre ensuite une parenthèse pour reprocher à Didon d'avoir deux poids, deux mesures : elle qui a dû fuir la Phénicie est désormais attachée à Carthage et à la Libye, pourquoi refuse-t-elle aux Troyens de s'établir en Ausonie (347-350). Puis, il reprend le fil de sa défense en rejetant la responsabilité du choix qu'il fait sur : $2^{\circ}$ son père Anchise qui lui apparaît chaque nuit en sommeil et dont l'image l'effraie (351-353) ; $3^{\circ}$ son fils Ascagne qu'il ne voudrait pas frustrer du royaume d'Hespérie et des terres qui lui sont destinées (354-355) ; $4^{\circ}$ enfin il raconte l'épiphanie de Mercure qui lui a transmis les ordres de Jupiter; il assure à Didon qu'il a vu le dieu dans toute sa splendeur à l'intérieur du palais et qu'il a entendu sa voix (356-359). Ces trois autorités qui cimentent sa décision de partir sont énumérées dans l'ordre croissant de l'importance qu'Énée et sans doute ses lecteurs contemporains y attachent : (1) la pietas due à son père ; (2) la pietas envers son fils et sa lignée ; (3) enfin et surtout la pietas envers les dieux.

10 La conclusion est brève et de nouveau péremptoire : il enjoint à Didon d'arrêter de les tourmenter l'un et l'autre de ses plaintes ${ }^{11}$; et il répète de manière lapidaire que ce n'est pas de sa propre initiative qu'il embarque pour l'Italie (360-361).

11 En somme, dans la première partie de son discours (333-344), Énée répond dans l'ordre aux différents points (dissimulation, mariage, attrait de l'Italie plus fort que celui de 
Troie) soulevés par Didon dans la première partie de son propre discours où elle a essayé de le raisonner. Par contre, il passe complètement sous silence la deuxième partie où elle s'est efforcée de l'émouvoir et de l'apitoyer. À ses querelae («plaintes »), à ses considérations d'ordre privé, il oppose les raisons contraignantes qui ont motivé son choix et qu'il n'appartient ni à lui ni à elle ( 360 : « desine meque tuis incendere teque querelis ", " cesse d'attiser de tes plaintes à la fois mon cœur et le tien ») de remettre en question.

\section{La réplique de Didon}

12 La réaction de Didon est enflammée ( accensa »): elle l'accuse d'insensibilité, de félonie, de cruauté (365-373); elle crie son désespoir, elle rappelle une fois encore comment elle a recueilli et sauvé les naufragés (373-375), elle rejette avec sarcasme sa prétention de ne faire qu'obéir à l'oracle d'Apollon et aux injonctions de Jupiter, ainsi que d'avoir vu et entendu Mercure (376-380). Elle le maudit et le voue aux pires supplices. Enfin, elle annonce qu'elle va se suicider et le poursuivre partout comme une ombre $\left(380-387^{12}\right)$.

\section{Aperçu des commentateurs modernes}

Depuis l'époque romantique, le discours d'Énée a profondément choqué les éditeurs. Ainsi, l'abbé Delille estima que « peut-être l'amant de Didon, prêt à la quitter, lui devait une réponse plus douce et plus galante, et des expressions plus vives de reconnaissance et de regret ${ }^{13}$. " Au début du xx siècle, Augustin Cartault s'offusqua du «ton glacial, inutilement blessant » de ce discours et il conclut : «Il faut bien croire que Virgile, en mettant dans la bouche d'Énée cette réponse déplorable et d'une incompréhension pénible, n'en a pas senti l'inconvenance et qu'il lui arrive parfois de manquer de goût ${ }^{14}$. » À la même époque, le grand latiniste Richard Heinze nota qu'aux yeux des modernes le comportement d'Énée doit apparaître froid et peu chevaleresque ${ }^{15}$. Plus près de nous, Jacques Perret note non sans embarras : « on ne croira pas qu'en une telle situation il (i.e. Virgile) ait voulu lui faire tenir des propos d'une vulgarité creuse, lui prêter de bonnes paroles ${ }^{16} »$ (t. 1, p. 187). Même embarras chez Gilbert Highet qui avance même l'hypothèse que Virgile a intentionnellement mis dans la bouche d'Énée un discours déplacé en l'occurrence ("Apparently Vergil intended it to be inadequate ${ }^{17} »$ ), sans préciser en quoi ce discours est déplacé et tout en reconnaissant aussitôt après qu'il est composé avec habileté.

14 Ces appréciations sont-elles le fait de lecteurs qui rétroprojettent sur des textes anciens des conceptions de leur propre époque et les jugent à l'aune de critères anachroniques ${ }^{18}$ ? Tel est d'ailleurs dans une large mesure également le cas de certaines tentatives psychologisantes modernes de défendre Énée contre ces critiques. Ainsi quand Eckard Lefèvre soutient, après d'autres, que le discours d'Énée reflète le conflit intérieur entre devoir ( " pietas ») et désir qui déchire de manière tragique le héros et qu'il surmonte de manière exemplaire ${ }^{19}$.

15 Il est étrange que, jusqu'à une date toute récente, peu de savants modernes aient jugé utile de recourir à une grille de lecture rhétorique de ces deux discours et de les étudier en détail selon les critères de la rhétorique avec laquelle Virgile s'était familiarisé au temps de sa jeunesse ${ }^{20}$. Et ce n'est que tout récemment que l'on a redécouvert 
l'importance des Interpretationes Vergilianae de Tiberius Claudius Donatus pour notre compréhension de la composition de l'Énéide ${ }^{21}$.

\section{Analyse rhétorique du discours d'Énée}

16 Dans la deuxième partie de cet article, je me propose de reprendre l'analyse du discours d'Énée et de l'approfondir à la lumière des annotations de Donat ${ }^{22}$ et d'autres commentateurs antiques, Servius et Servius auctus (Scholia Danielis ${ }^{23}$ ) ainsi que des remarques de plusieurs éditeurs et commentateurs $\mathrm{du}_{\mathrm{XVI}}^{\mathrm{e}}$ au $\mathrm{XVIII}^{\mathrm{e}}$ siècle : Philippe Melanchthon ${ }^{24}$, Hortensius Lambertus ${ }^{25}$, Nascimbene Nascimbeni ${ }^{26}$, Jules-César Scaliger ${ }^{27}$, Melchior Laubanus ${ }^{28}$, Jacobus Pontanus ${ }^{29}$, Juan Luis de La Cerda ${ }^{30}$, Charles de La Rue ${ }^{31}$ et Marco Antonio Ferrazzi ${ }^{32}$.

\section{A. Les vers $333-339$}

Donat commence par évaluer l'impact du discours de Didon. Il ne distingue pas explicitement entre l'effet du discours sur le lecteur d'une part, sur Énée d'autre part. Quand il affirme que «Didon a fait valoir à l'appui de sa cause des arguments justes, humains et susceptibles d'éveiller la pitié qui auraient pu et auraient dû fléchir Énée ${ }^{33}$ ", il parle évidemment du point de vue du lecteur. Ce même regard de lecteur lui fait dire ensuite qu'il était évident («constabat»: mais pour qui ?) qu'Énée avait honte d'« endosser, malgré lui et sur ordre de Jupiter, le rôle d'un homme ingrat, imposteur et déserteur" ("qui ingrati, qui fallacis, qui fugitiui personam contra institutum suum Ioue cogente suscepisset »). Le lecteur ancien n'est donc pas insensible à la dimension humaine de cette rupture brutale, mais il est plus compréhensif à l'égard du choix d'Énée, sans doute parce que ce choix obéit à des valeurs auxquelles il souscrit plus volontiers que le lecteur moderne. S'en tenir aux ordres célestes, c'est agir « reuerenter ", « avec scrupule ». En conséquence, selon Donat, ne pas céder aux demandes et aux larmes de Didon, mais tenir ferme son regard, ce n'est pas faire preuve d'inhumanité, c'est avoir sous les yeux le seul Jupiter et «ses ordres auxquels il était impossible de rester indifférent» («a cuius praeceptis moueri non poterat»). Selon Donat, il n'y a pas lieu d'accuser Énée d'inhumanité, puisqu'il n'est pas resté insensible, le poète précisant qu'il a fait de grands efforts pour étouffer sa peine au plus profond de lui-même. Énée a simplement respecté la hiérarchie des valeurs et fait " passer les désirs humains après les ordres divins », " postponens humana caelestibus ${ }^{34}$.»

18 Au vers 331 («tandem pauca refert»), Donat ne s'étonne pas de voir Virgile qualifier de "pauca» un discours en fait plus long que le discours auquel il répond. Ici encore, il explique l'impression subjective du lecteur : Didon a parlé longuement et cela en accord avec sa situation; elle avait été surprise par un malheur soudain et il était dans son intérêt de parler beaucoup pour retarder celui qui avait hâte de partir ${ }^{35}$. Énée se devait au contraire, toujours selon Donat, de répondre brièvement, soit pour ne pas s'attarder sur l'accusation gênante de félonie, soit pour ne pas perdre de temps avec du bavardage inutile à un moment où il était obligé de partir. Donat donne ainsi une explication fonctionnelle de la breuitas, qui n'est pas réelle, mais au service de l'ethos de l'orateur ${ }^{36}$.

19 Le fait que le discours ne commence pas au début du vers, mais à la coupe penthémimère n'a guère intrigué les commentateurs antiques ou modernes. Ce n'est que récemment que $G$. Highet a essayé de trouver une explication: le poète veut 
marquer une pause avant les premiers mots, une pause qui exprime l'effort de maitrise de soi chez Énée, laquelle se manifeste aussi à travers le calme sérieux du début du discours ${ }^{37}$.

En abordant le discours proprement dit, Donat s'arrête sur le choix du thème exordial (Énée reconnaît les mérites de la reine à son égard; il se souviendra toujours avec plaisir d'elle). Être accusé d'ingratitude est particulièrement odieux, et Énée réfute donc en priorité cette accusation. Tout discours de Virgile ayant, aux yeux de Donat, une valeur exemplaire ${ }^{38}$, ce dernier attire l'attention (de son fils, dédicataire de son livre) sur le fait que ce discours illustre la règle que, confronté à une avalanche de griefs, l'orateur doit commencer par en réfuter les plus solides, afin de ne pas avoir l'air de perdre son temps avec des futilités par peur de reproches graves (p. 402, 22-27).

Autre remarque subtile de Donat, qui montre bien à quel point les commentateurs anciens étaient sensibles à l'ethos de l'orateur : en accordant à Didon qu'elle avait bien mérité de lui et des siens (chose évidente qu'Énée aurait été bien incapable de nier), il parle d'elle comme un personnage haut placé parle d'un subalterne méritant (p. 403, 1 : "sic locutus est quasi potiori inferior obsecuta uideatur", " il parla comme si elle devait apparaître comme une subalterne qui a obéi à son supérieur »). Cette explication semble être contredite par le choix du terme regina dont Énée se sert pour interpeller Didon et que Donat ne commente malheureusement pas. Or, dans la remarque suivante, Donat note qu'Énée fait bien la distinction entre les largesses que lui et les siens ont reçues de Didon ${ }^{39}$ et la personne même de cette dernière (p. 403, $6:$ « recolens nominis tui beneficiorumque memoriam : ista ergo habeant separatam rationem suam ", " en ressuscitant le souvenir de ton nom et de tes largesses; ces dernières sont donc comptabilisées à part »). Donat a ainsi bien vu la différence qu'Énée fait désormais entre le rôle public et le personnage privé, tant dans son propre chef que dans celui de Didon: la femme aimée a droit à un remerciement affectueux (Elissae) et appuyé (épanaphore de «dum »), la reine à des remerciements plus officiels de la part de celui qui est investi par les dieux de la mission de préparer la fondation de Rome et qui ne peut donc être sur un pied d'égalité avec la fondatrice de Carthage. Bien qu'il ne le dise pas explicitement, Donat semble avoir bien senti que le titre de regina n'est pas seulement donné ici à Didon pour la flatter (" beneuolentiae causa ${ }^{40} »$ ) ni le choix d'Elissa fait uniquement pour des raisons métriques ${ }^{41}$, mais qu'ils permettent à Énée de mettre l'un et l'autre à sa place, de renvoyer son interlocutrice à son rôle public et de rompre ainsi avec leur intimité qui appartient désormais au passé et n'est plus qu'un souvenir ${ }^{42}$. Dans ce sens, il est correct de dire que cet exorde (Donat évite le terme) est a nostra et aduersarii persona; l'orateur y redéfinit l'ethos qu'il souhaite imposer à son auditrice. À propos de l'affirmation qui suit et clôt l'exorde (337: " pro re pauca loquor », "sur le fond, j'ai peu à dire »), Donat en explicite un des sous-entendus : quand la vérité saute aux yeux, nul besoin de longues paroles (p. 403,9: "ueritas enim multiloquio non adseritur »), mais il ne se rappelle apparemment plus que le breuiloquium est aussi une preuve de détermination, comme il l'avait suggéré plus haut (p.402,20). Autre sous-entendu judicieusement explicité par Donat : le breuiloquium aussi permet à Énée de prendre ses distances avec Didon (p. 403,10: "neque ego, ut tu, commorabor in plurimis ", "et, contrairement à toi, je ne m'attarderai pas à un long discours »).

Sans avoir explicitement délimité un exorde dans ce discours, Donat signale ensuite le début de la réfutation proprement dite: "incipit iam obiecta purgare uel refutando excludere ", « il commence maintenant à se justifier des accusations soit à les réfuter et 
rejeter » (p. 403,11). Ici encore, sans recourir à la terminologie consacrée, Donat voit à l'œuvre deux lignes de défense distinctes : l'une consiste à justifier (" purgare »), l'autre à rejeter ( refutando excludere ») la conduite qui lui a été reprochée. Les termes techniques se trouvent chez Servius ${ }^{43}$ : «La discussion est parfaite : il s'y justifie des reproches en repoussant l'accusation d'être un ingrat ${ }^{44}$, et en même temps il a recours à l'état de cause véniel, rejetant la faute de son départ sur la volonté des dieux. Il utilise aussi la définition, car il justifie la fuite qu'on lui reproche en la qualifiant de départ. » À noter que, dans leurs aperçus anticipés respectifs, tant Servius que Donat décrivent d'abord la ligne de défense utilisée dans la deuxième partie (345-359), puis seulement celle de la première partie (337-339). Selon Servius, l'essentiel de la défense est plaidé selon l'état de cause véniel («status causae uenialis »), la première partie selon l'état de cause définitoire («status causae finitionis »).

L'expression "status uenialis " n'apparaît que chez les rhéteurs tardifs ${ }^{45}$. Cet état de cause correspond à une subdivision de l'état de cause de la qualification ${ }^{46}$ judiciaire (" constitutio generalis iuridicialis, pars adsumptiuua»), appelée concessio, " $\operatorname{aveu}^{47} »$, qui consiste à justifier non pas l'acte commis en soi, mais à demander pardon ( «ut ignoscatur ", d'où l'appellation status uenialis), et cela de deux manières dont l'une est la purgatio (excuse) qui consiste à justifier l'intention (« uoluntas») de l'accusé, en faisant valoir son ignorance, le hasard malheureux ou une force majeure («imprudentia, casus, necessitudo $\left.{ }^{48} »\right)$.

Cette interprétation est reprise par N. Nascimbeni qui note en marge du vers 333 : « état de la qualification judiciaire, par l'aveu du fait mis en cause, parce qu'il transfère la cause de son départ à la volonté de Jupiter ${ }^{49}$. » Auparavant, Ph. Melanchthon avait déjà parlé de « disculpation par transfert. Il rejette en effet toute la faute sur les dieux et le destin ${ }^{50}$. » Une variante se trouve chez J. C. Scaliger qui semble voir ici à l'œuvre la troisième subdivision du status qualitatis, à savoir le report de l'accusation (remotio criminis), qui consiste à rejeter la responsabilité de l'acte commis sur une autre personne ou une autre chose ${ }^{51}$.

À une première lecture des vers 337-338 («Neque ego hanc abscondere furto / speraui, ne finge, fugam ", "je n'ai pas espéré cacher ceci subrepticement comme une fuite, ne te l'imagine pas »), on a l'impression qu'Énée nie simplement, mais catégoriquement l'idée d'avoir espéré s'enfuir en catimini de Carthage, recourant ainsi à l'état de cause conjectural (à «je n'ai pas fait » correspond en l'occurrence "je n'ai pas espéré5 ${ }^{52}$ ). Or, selon Donat, il conteste la qualification (définition) que Didon a donnée de son projet, recourant donc à l'état de cause définitoire ("status definitiuus $\left.{ }^{53} »\right)$ : J'ai préparé non pas ma fuite, mais mon départ en bateau («non fugam, sed nauigationem paraui $\left.{ }^{54} »\right)$. Même ligne de défense pour nier qu'il y ait eu un mariage en bonne et due forme. Paraphrasant les propos d'Énée pour mieux les élucider, Donat lui fait dire : «Je me suis uni à toi, dit-il, mais impossible d'appeler cela mariage ; en effet, toute union d'une femme et d'un homme ne crée pas de mariage. Ce que nous avons fait porte un autre $n^{n o m}{ }^{55}$. " Seruius auctus précise : " est quasi status finis latens : quid sint legitimae nuptiae », « il y a là comme un état de cause définitoire latent ${ }^{56}$. » Dans le deuxième hémistiche du vers 339 (" aut haec in foedera ueni », « je ne suis pas venu pour une telle alliance »), Énée semble rappeler que sa liaison avec Didon était une péripétie non prévue dans sa mission $^{57}$. La brutalité de cette mise au point est cohérente avec cette remarque d'auteur où le poète avait relevé que Didon se baignait d'illusions au soir de son union 
avec Énée (171-172) : «Ce n'est plus un amour clandestin qu'elle envisage, / elle parle de mariage et de ce nom elle voile sa faute ${ }^{58}$. »

\section{B. Les vers $340-347 a$}

Énée se défend ensuite (340-347) de montrer plus d'empressement pour prendre le large vers une destination inconnue qu'il n'en montrerait pour retourner à Troie si elle était encore debout. En réponse à ce reproche, il entame de manière encore discrète la ligne de défense qu'il gardera jusqu'à la fin du discours et qui consiste non pas à nier ce qu'on lui reproche, mais à le justifier par son obéissance à des valeurs supérieures. $1^{\circ}$ «Si j'étais libre de mon destin, je me trouverais à Troie, auprès des restes des miens, j'aurais déjà reconstruit le palais de Priam et la ville de Pergame». Donat explicite: "Ce que je suis en train de faire, dit-il, c'est-à-dire appareiller, n'est pas de ma volonté et je ne suis pas libre de vivre dans un lieu de mon choix; si tel était le cas, il vaudrait mieux pour moi de rester au milieu des cendres de ma patrie, etc. ${ }^{59}$ ». $2^{\circ}$ " Mais l'oracle d'Apollon m'ordonne de gagner l'Italie »; $3^{\circ}$ « donc, c'est là que se trouvent mon cœur et ma patrie ».

Nous avons vu plus haut que Servius, suivi des commentateurs modernes (Nascimbeni), a qualifié cette ligne de défense d'état de cause véniel. Seulement, la pertinence de cette analyse peut être mise en doute. Dans l'aveu (concessio), l'accusé reconnaît le caractère répréhensible de son acte et cherche à le justifier en faisant valoir des excuses, en l'occurrence la volonté des dieux ${ }^{60}$. Or, il est plus que douteux qu'Énée ait accepté de concéder que son départ soit en lui-même condamnable. S'il présente son départ comme une décision juste et nécessaire parce qu'il est voulu des dieux et parce qu'il lui permet d'honorer ses obligations (pietas) à l'égard de son père, de son fils et des dieux, il ne se décharge pas pour autant de sa responsabilité sur les dieux, son père et son fils, mais soutient que sa décision est juste parce que conforme à ses devoirs. Or, la ligne de défense qui plaide l'équité de l'acte même est la qualitas iuridicialis absolutae, qui contient en elle-même sa justification ${ }^{61}$.

Par ailleurs, à part pour le grief relatif à la dissimulatio observée dans les préparatifs du départ, le débat porte non sur des faits passés, comme il est de coutume dans le genre judiciaire, mais sur des faits à venir. Or, les rhéteurs ont prévu un état de cause à part pour les débats portant sur un acte à venir et relevant de ce fait davantage du genre délibératif que du genre judiciaire, à savoir l'état de cause pragmatique ${ }^{62}$.

Melchior Laubanus a décortiqué ces huit vers 340-347 comme un long syllogisme hypothétique ${ }^{63}$. La majeure implicite serait : «si je ne suis pas maître des mes actes, je ne suis pas coupable ». La conclusion est déduite de la preuve de la mineure (« or, je ne suis pas maitre de mes actes ") qui est développée sous forme d'un prosyllogisme de la deuxième figure (dite modus tollens) dont la majeure est « Si j'étais maître de mes actes, je reconstruirais Troie ", la mineure ("Or, je ne reconstruis pas Troie») et la conclusion étant sous-entendues. À la place de la mineure, Virgile amplifie la cause positive qui pousse Énée à partir, à savoir l'oracle d'Apollon, et la conclusion prend la forme inattendue d'un épiphonème : «Là est mon affection, là ma patrie » (« hic amor, haec patria est $\left.{ }^{64} »\right)$. 


\section{Les vers $347 \mathrm{~b}-350$}

30 Avant de continuer dans l'énumération des « auctoritates " responsables de son départ, Énée ouvre une parenthèse pour reprocher à Didon de lui en vouloir de partir pour l'Italie. À l'aide d'un parallèle (collatio) entre la situation de Didon et la sienne (sous forme de syllogisme de la première figure), il dénie à Didon tout droit de lui refuser de partir : si elle, une Phénicienne, s'est établie à Carthage, les Troyens ont eux aussi reçu des dieux le droit ( $\left(f a s^{65} »\right)$ de chercher un royaume étranger ${ }^{66}$. Le deuxième membre de la majeure prend la forme d'une question rhétorique exprimant l'indignation ${ }^{67}$ : « quae tandem [...] inuidia est ? » Non content de se justifier, Énée attaque désormais Didon et lui reproche ${ }^{68}$ de vouloir par jalousie le frustrer d'un bien dont elle jouit sans hésitation. Scaliger note ici fort judicieusement qu'Énée reproche à Didon non pas de souhaiter le garder auprès d'elle, mais d'entendre interdire aux Troyens d'aller s'installer là-bas ${ }^{69}$.

31 Apparemment, aucun commentateur ne semble s'être aperçu que cette comparatio parium ("parallèle ») est également au service de la remotio criminis ou " rejet de la responsabilité » ( $3^{\mathrm{e}}$ variante de l'«état de cause de la qualification empruntée ») : l'exemple de Didon, à l'égal de l'oracle d'Apollon et des autres autorités citées plus loin, a autorisé Énée à agir comme il le fera. Donat note d'ailleurs dans ce sens : «nullus autem melius et robustius uincitur quam qui proprio superatur exemplo", " on ne l'emporte sur personne mieux et plus massivement que sur celui qui doit céder à son propre exemple.»

\section{Les vers 351-359}

L'énumération des trois dernières « autorités » sur lesquelles Énée appuie sa demande est interprétée par N. Nascimbeni comme une amplification à partir de preuves extratechniques ${ }^{70}$. Comme les commentateurs antiques, il insiste sur le soin apporté à assurer la vraisemblance des apparitions nocturnes d'Anchise en décrivant avec un luxe de détails les circonstances de temps ${ }^{71}$. Même souci de l'«euidentia» dans la description de l'épiphanie de Mercure ( ipse deum uidi, in manifesto lumine ${ }^{72}$, intrantem muros; hausi uocem his auribus ", " de mes propres yeux j'ai vu le dieu, dans une lumière rayonnante, entrer dans ces murs; j'ai imprégné mes oreilles de sa voix »), où, face à une auditrice sceptique, il souhaite faire revivre la scène dont il a été témoin et dont il atteste solennellement la véracité ${ }^{73}$.

\section{E. Les vers $360-361$}

La péroraison est péremptoire, sous forme d'un ordre donné à Didon, lui intimant de cesser de jeter de l'huile sur le feu avec des plaintes («querelis», 360), au motif sousentendu qu'elles seront inutiles ${ }^{74}$. En fait, il justifie son ordre dans une sorte de récapitulation de la dernière partie de son argumentation ("Italiam non sponte sequor ${ }^{75}$ $»)$.

«Italiam non sponte sequor » (vers 361) ne veut pas dire qu'il part contre son gré, mais qu'il ne part pas de sa propre initiative ${ }^{76}$; il lui fallait un rappel à l'ordre de la part de Jupiter (331: «Iouis monitis »). Même si ce départ n'est pas de son propre choix, il suit de toute son âme la décision du destin et des dieux: "Ardet abire fuga dulcesque 
relinquere terras ", "il brûle de prendre la fuite et de partir, de quitter ces douces terres »(281). Le vers 361 est incomplet: était-ce voulu par le poète ou l'effet malheureux de l'état inachevé de l'Énéide? Servius auctus estime que Virgile a intentionnellement laissé le vers incomplet: "et oratorie ibi finiuit ubi uis argumenti consistit ", " et, tel un bon orateur, il s'est arrêté à son argument le plus fort ${ }^{77}$ ». Mais l'explication que le même scoliaste donne du participe présent "dicentem " au vers suivant (362) semble suggérer qu'il imagine que Didon a interrompu Énée et que le vers incomplet signale que le héros n'avait pas encore entièrement terminé son discours ${ }^{78}$.

L'étude qui précède devait simplement illustrer comment l'analyse rhétorique, pratiquée avec discrétion et élégance par un Donat, avec érudition par les savants humanistes et jésuites, permet de comprendre que chaque discours virgilien est le fruit de la mise en œuvre consciente et consciencieuse de règles précises, bien connues des premiers lecteurs, et nous révèle « in poeta summum oratorem », « sous le poète, un très grand rhéteur »; et, dans son magnifique poème, «artem dicendi plenissimam ${ }^{79}$ », « un art de la parole accompli ». Comme le dit Macrobe ${ }^{80}$ :

tous, en parlant entre eux à voix basse, reconnaissaient unanimement que Virgile devait être considéré comme non moins un orateur qu'un poète, puisqu'on trouvait chez lui à la fois une profonde connaissance de l'art oratoire et une application stricte des règles de la rhétorique.

\section{NOTES}

1. A. Wlosok, "Vergils Didotragödie : ein Beitrag zum Problem des Tragischen in der Aeneis", dans H. Görgemanns-E. A. Schmidt éd., Studien zum antiken Epos, Meisenheim a.Gl., 1976, p. 228-250 ; R. C. Monti, The Dido episode and the Aeneid: Roman social and political values in the epic, Leiden, 1981.

2. R. J. Starr, «Aeneas the rhetorician : Aeneid IV, 279-295 », dans Latomus 62 (2003), p. 36-46.

3. Pour une analyse détaillée de ce discours, $c f$. M. Erdmann, Überredende Reden in Vergils Aeneis, Frankfurt a.M.-Berlin [etc.], 2000, p.128-178. Cf. aussi E. M. Ingram, « Rhetorical elements in Homer's embassy and Vergil's Dido", Senior Capstone projects ; paper 71/2012 p. 8-11 (http:// digitalwindow.vassar.edu/senior_capstone/71).

4. 4.328-329 : "si quis mihi paruulus aula / luderet Aeneas ", "si dans mon palais jouait un tout petit Énée à moi ». Le choix de paruulus, unique occurrence d'un adjectif diminutif dans l'Énéide (cf. R. G. Austin éd., P. Vergili Maronis Aeneidos liber quartus, Oxford 1963, p. 104), est révélateur du changement de ton au fil du discours: inquisitoriale dans l'exorde, Didon se fait désormais suppliante et essaie d'apitoyer Énée.

5. Le sens de cura est ambigu : chagrin d'amour ou peine en général? Cf. déjà Servius auct. ad 332, p. 523,5: "curam uel simpliciter accipe, uel amorem ", "comprends curam soit dans son sens premier, soit dans le sens d'amour ». Si cura est synonyme de amor, c'est ici le premier passage où Virgile évoque explicitement l'amour d'Énée pour Didon ( $c f$. S. Farron, « The Aeneas-Dido episode as an attack on Aeneas' mission and Rome ", dans Greece and Rome 27, 1980, p. 35). À ce vers fera écho le vers 395 : «multa gemens magnoque animum labefactus amore ", " gémissant longuement et secoué par son grand amour».—《Premebat» («il étouffait») a certainement ici une valeur conative (« il tâchait d'étouffer »). 
6. Cf. E. Lefèvre, "Aeneas' Antwort an Dido", dans Wiener Studien n.s. 8 (1974), p. 99-115; F. Loretto, « Aeneas zwischen Liebe und Pflicht : ein Beitrag zur Interpretation von Vergil, Aeneis 4, vv. 331-361", dans Der altsprachliche Unterricht, 13/5 (1970), p. 27-40, et surtout D. Feeney, « The taciturnity of Aeneas ", dans Classical Quaterly 33 (1983), p. 204-219.

7. Une remarque de Servius auct., ad loc. (p. 523,29: « pauca au sens premier, c'est-à-dire compte tenu de l'importance de l'affaire ») conduit certains éditeurs à comprendre « pro re » dans le sens de " $\mathrm{au}$ vu des circonstances». Je préfère interpréter "pro re » dans le sens de "pro causa ", «pour ma défense » ou « sur le fond ». Cf. D. Feeney, op. cit., p. 206.

8. Aux vers 337-338. Cf. cependant notre analyse infra, § 25.

9. Selon D. Feeney, op. cit., p. 208, le choix de fingere ( inventer ») implique que les accusations sont forgées de toutes pièces. Virgile emploie fingere dans ce sens (par exemple Aen. 11.406), mais il l'utilise aussi dans le sens plus neutre de excogitare, imaginari, ce qui semble le cas en l'occurrence, $c f$. Thesaurus Linguae Latinae, VI.1, 775.30 (où il est renvoyé à notre passage ainsi qu'à Aen. $7.438 ; 8.42$; 11.364). Pour l'impératif, cf. R. F. Thomas, Reading Virgil and his texts, Ann Arbor, 1999 , p. 224-226.

10. Énée sous-entend ici le principe qu'Ulpien formulera plus tard comme suit (Digeste, 35.1.15): «nuptias enim non concubitus, sed consensus facit », " en effet c'est non pas la copulation, mais le consentement qui crée le mariage."

11. Selon M. Helzle (dans Der Stil ist der Mensch : Redner und Reden im römischen Epos, Stuttgart, B. G. Teubner, 1996, p. 54), querela a ici un sens érotique («plainte amoureuse »). Toutefois, D. Feeney (article cité, p. 209) montre, à l'aide de passages du De oratore de Cicéron, que tant incendere que querela appartiennent au vocabulaire de la rhétorique et servent à décrire le style pathétique, qui était justement celui du discours de Didon.

12. R. D. Williams, "Dido's reply to Aeneas (Aen. 4.362-387)", dans H. Bardon-R. Verdière éd., Vergiliana : recherches sur Virgile, Leiden, 1971, p. 421-442.

13. J. Delille (trad.), L'Énéide t. 2, Paris, 1804, p. 124. Déjà le P. de La Rue (1643-1725), éditeur de Virgile dans la collection ad usum Delphini (Paris, 1682), p.421, avait jugé bon de noter: " Fatendum tamen orationem illam, si deorum iussa excipias, sane esse subfrigidam », " il faut pourtant reconnaitre que, si l'on excepte les ordres divins, ce discours est sûrement quelque peu froid». En fait, des critiques du rôle que Virgile prête ici à Énée se trouvent déjà au Moyen Âge. $C f$. Chaucer, The house of Fame, v. 267 : «For he to hir a traytour was " ("Car il l'a trahie »); id., The legend of good women, v. 1325-1326: «And as a traytour forth he gan to sayle / Toward the large contre of Ytayle » («Et le traître fit voile / Vers la grande terre d'Italie »). Références chez A. Wlosok, Der Held als Ärgernis : Vergils Aeneas, dans Würzburger Jahrbücher, 8 (1982), p. 15.

14. A. Cartault, L'art de Virgile dans l'Énéide, Paris, 1926, p. 319-320. Tout aussi péremptoire le jugement de l'auteur du commentaire anglais le plus influent de l'époque, T. E. Page éd., The Aeneid of Virgil. Books I-VI, London, 1894, p. 370 : «Not all Virgil's art can make the figure of Aeneas here appear other than despicable. His conduct had been vile» («Tout l'art de Virgile ne peut faire qu'ici la figure d'Énée n'apparaisse pour autre chose que méprisable. Sa conduite a été vile. »).

15. R. Heinze, Virgils epische Technik, Leipzig-Berlin, 3e éd. 1915, p. 123-124, n. 1 (« Modernem Gefühl erscheint das Benehmen des Aeneas leicht herzlos und unritterlich »). Cf. aussi V. Pöschl, « Dido und Aeneas ", dans Festschrift Karl Vretska zum 70. Geburtstag, Heidelberg, 1970, p. 148-173 (p. 160) : « Aber auch wenn man zugibt, dass Aeneas sich zur Härte zwingt, um nicht schwach zu werden, so hat doch die kalte Herzlosigkeit seiner Worte dennoch etwas Verletzendes und Abstossendes und lässt nicht nur bei Dido, sondern auch bei jedem Leser ein Gefühl der Befremdung und des Missbehagens zurück." Nous traduisons : « Même si l'on admet qu'Énée se force à être dur, pour ne pas céder, la froideur et le manque de chaleur de ses paroles ont néanmoins quelque chose de blessant et de répugnant et suscitent non seulement chez Didon, mais chez tout lecteur un réel désagrément et malaise. »

16. J. Perret éd., Virgile, L'Énéide, livres I-IV, Paris, Les Belles Lettres, C.U.F., $2^{\mathrm{e}}$ éd. 1999, p. 187. 
17. G. Highet, The speeches in Vergil's Aeneid, Princeton NJ, 1972, p. 75. Cf. déjà T. E. Page, op. cit., p. 370 « Virgil is conscious, however, that as a reply it is inadequate " ( Virgile est néanmoins conscient que comme réplique c'est inadéquat »).

18. Cf. Montesquieu, L'Esprit des lois, livre 30, chap. 14: "Transporter dans des siècles reculés toutes les idées du siècle où l'on vit, c'est des sources de l'erreur celle qui est la plus féconde ».

19. E. Lefèvre, article cité. Plus convaincant me paraît l'essai récent de S. M. Braund, «Speech, silence and personality: the case of Aeneas and Dido », dans Proceedings of the Virgil Society 23 (1998), p. 129-147. Celle-ci interprète ce conflit à la lumière de la théorie des quatre personae ( « rôles ») de Cicéron : la troisième persona, celle qui est imposée par les circonstances extérieures (Cicéron, Les Devoirs ou De Officiis, 1.115), est respectivement celle d'un dux et d'une regina, donc d'un homme public ; à un moment donné, les deux se sont laissés aller à leur inclinaison privée, et il y eut conflit entre la troisième persona (celle qui leur est imposée) et la quatrième (celle qu'ils choisissent selon leur volonté ; Cicéron, Off., ibid.) : si Énée se ressaisit en reprenant la persona de $d u x$, faisant coïncider troisième et quatrième persona en lui, Didon refuse de renoncer au rôle qu'elle s'est choisi et qui l'empêche de maintenir son rôle public. Cf. déjà S. F. Wiltshire, Public and private in Vergil's Aeneid, Amherst, Univ. of Massachusetts Press, 1989, qui pensait que Didon confondait rôle politique (public) et rôle privé, alors qu'Énée reprenait à un moment donné conscience de la nécessaire séparation des deux rôles.

20. Très significative à cet égard est une remarque de R. Heinze, op. cit., p. 425-426: «Wie Aeneas seinerseits die Vorwürfe einen nach dem andern kurz zurückweist, um dann ausführlich und Punkt für Punkt darzulegen, dass seine Abfahrt keine freiwillige sei, das brauche ich nicht zu zeigen." Nous traduisons : «Inutile de montrer comment Énée, de son côté, réfute brièvement un à un chacun des reproches, pour expliquer ensuite de manière détaillée et dans l'ordre que son départ n'est pas volontaire ». De même G. Highet, op. cit., p. 12-13, n'a que du mépris pour la seule tentative moderne d'expliquer les discours de Virgile systématiquement à l'aide des règles de la rhétorique ancienne (K. Billmayer, Rhetorische Studien zu den Reden in Vergils Aeneis, Diss. Würzburg, 1932) : supposer que les discours obéissent à ces règles est, selon Highet, une grave erreur ( $a$ serious misconception »). Pour un jugement plus équilibré sur le travail de K. Billmayer, cf. M. Erdmann, op. cit., p. 5.

21. Le mérite de la réhabilitation de Donat revient à $\mathrm{M}$. Squillante Saccone, Le Interpretationes Vergilianae di Tiberio Claudio Donato, Napoli, 1985. Cf. aussi R. J. Starr, « An epic of praise : Tiberius Claudius Donatus and Vergil's Aeneid», dans Classical Antiquity 11 (1992), p. 159-174 ; G. Moretti, "The poet in court : judiciary model in literary criticism : the case of Tiberius Claudius Donatus ", dans A. Pennacini dir., Studi di retorica oggi in Italia 1997, Bologna, 1998, p. 59-71; et surtout dans notre perspective L. Pirovano, Le Interpretationes Vergilianae di Tiberio Claudio Donato : problemi di retorica, Roma, 2006. Pour l'épisode avec Didon, cf. aussi M. Gioseffi, « Nusquam sic uitia amori: Tiberio Claudio Donato di fronte a Didone », dans F. Conca éd., Riccordando Raffaele Cantarella: miscellanea di studi, Milano, 1999, p. 137-163.

22. Cité d'après l'éd. de H. Georgii (Leipzig, Teubner, 1905).

23. Cités d'après l'éd. de G. Thilo-H. Hagen (Leipzig, Teubner, 1881).

24. Cité d'après l'éd. Pub. Vergilii Maronis opera, Lugduni, 1556. La première édition de l'Enarratio Aeneidos Virgilii remonte à 1530.

25. Cité d'après l'éd. Lamberti Hortensii Montfortii enarrationes doctiss. atque utiliss. in XII libros P. Virgilii Maronis Aeneidos. His accessit Nascimbaeni Nascimbaenii in priorem P. Virgilii Maronis epopoeiae partem, id est, in sex primos Aeneidos libros erudita et perelegans explanatio, Basileae, 1577 ( $1^{\text {re }}$ éd. avec les notes du seul Lambertus Hortensius, Bâle, 1559). Pour l'auteur, cf. A. J. Van der Aa, Biographisch woordenboek der Nederlanden, Deel 8/2, Haarlem, 1867, p. 1284-1288; Jacob Cornelis van Slee, «Hortensius », dans Allgemeine Deutsche Biographie, vol. 13 (1881), p. 164-165. Recteur de l'école de Narden, Lambert van den Hove est mort en 1574, probablement à l'âge de 74 
ans. Il est l'auteur de nombreux travaux d'histoire contemporaine et de philologie (Aristophane, Virgile, Lucain).

26. Pour l'auteur, $c f$. en dernier lieu R. Seferovič, « Foreign teacher and humanist : Nascimbene Nascimbeni on rhetoric in Dubrovnik», dans Dubrovnik annals 14 (2010), p. 99-141. Originaire de Ferrare, Nascimbeni avait enseigné dans sa ville natale et à Reggio Emilia, avant de devenir, dans les années 1560, recteur de l'école de Dubrovnik. Il publia notamment un ouvrage intitulé Grammatilogia (Bologne, 1555), une édition commentée du De l'invention de Cicéron (Venise, 1564) et un commentaire des six premiers livres de l'Énéide (Bâle, 1577).

27. Consulté dans l'éd. moderne de L. Deitz, Iulius Caesar Scaliger, Poetices libri septem. Sieben Bücher über die Dichtkunst, vol. 2 (Buch 3, Kapitel 1-94), Stuttgart-Bad Cannstatt, 1994.

28. M. Mel. Laubani Archetyporum analyticorum ad integram P. Virg. Maronis Aeneidem libri septem, quorum primis quatuor totidem Aeneidos perpetua luculentaque singulorum non tam uersuum quam membrorum minutissimorum analysi logica, grammatica, rhetorica, mythologica, rituali [...] ad oculum repraesentantur, Lignicii Silesiorum, 1610. Pour l'auteur, professeur à Sprottau (Silésie), Goldberg, Dantzig et Brieg, cf. Chr. Jöcher, Compendiöses Gelehrten-Lexicon, Leipzig, 1726, col. 1515-1516.

29. Consulté dans l'éd. Symbolarum libri XVII quibus P. Virgilii Maronis Bucolica, Georgica, Aeneis ex probatissimis auctoribus declarantur, comparantur, illustrantur, Augustae Vindelicorum, 1609 (1 ${ }^{\text {re éd. }}$ 1599). Sur le jésuite Jacob Spannmüller (1542-1626), cf. De Backer-Sommervogel, Bibliothèque de la Compagnie de Jésus, t. 6, Bruxelles-Paris, 1895, col. 1007-1019; B. Bauer, Pontanus, Jacobus, dans Neue Deutsche Biographie vol. 20 (2001), p. 615-616.

30. Consulté dans l'éd. P. Virgilii Maronis Aeneidos libri sex priores / argumentis, explicationibus et notis illustrati a Ioanne Ludovico de La Cerda, Coloniae Agrippinae, 1628 ( $1^{\text {re }}$ éd. Lugduni, 1612). Sur le jésuite de La Cerda (1558-1643), cf. De Backer-Sommervogel, op. cit., t. 2, 1891, col. 984-99; A. Laird, "Juan Luis de La Cerda and the predicament of commentary », dans R. K. Gibson-Chr. Shuttleworth Kraus éd., The classical commentary: histories, practices, theory, Leiden, 2002, p. 171-203.

31. Cité d'après P. Virgilii Maronis opera / interpretatione et notis illustrauit Carolus Ruaeus Soc. Jesu, Paris, 1682 (2 éd.). Sur le P. de La Rue (1643-1725), cf. De Backer-Sommervogel, op. cit., t. 7, 1896, col. 290-307; sur la collection dans laquelle paraissait son commentaire, $c f$. C. Volpilhac-Auger dir., La collection Ad usum Delphini : l'Antiquité au miroir du Grand Siècle, Grenoble, 2000.

32. Le commentaire de Marco Antonio Ferrazzi (Ferracci, Ferratius ; 1660/61-1748), préfet du séminaire de Padoue, est cité d'après l'édition anonyme Exercitationes rhetoricae in praecipuas P. Vergilii Maronis orationes quae in Aeneidum libris leguntur, imprimée à la suite du vol. 2 d'une réimpression de l'édition du $\mathrm{P}$. de La Rue parue en 1735 à Munich.

33. Donat, p. 401, 20 : «Dido pro causa sua adlegans iusta, humana, miseranda, quae Aeneae mentem flectere potuissent et deberent. "

34. Ibid., p. 402, 1-6. Suit un développement sur le choix par le poète du mot monitum ( avertissement ») au lieu de iussum («ordre ») : Énée ne reçoit pas d'ordre nouveau, mais les dieux lui rappellent sa mission arrêtée une fois pour toutes par le destin : se mettre au service de la conquête de l'Italie destinée à lui et à ses descendants, et non pas de Carthage l'ennemie.

35. Ibid., p. 402,16 : «quia Dido multa dixerat et post moras prolixas tacuerat. Hoc enim conueniebat ei quam repentinum turbauerat malum et ei quae plurimis utilia suis partibus extorquere nitebatur", «puisque Didon avait parlé beaucoup et ne s'était tue qu'au terme d'un discours prolixe et dilatoire. Voilà ce qui convenait parfaitement à une personne qui avait été ébranlée par un malheur soudain et s'efforçait par tous les moyens et frénétiquement à parvenir à ses fins. "

36. L'ethos de la concision est bien compris par Servius ad 1.561: «notandum regum esse breuiloquium, ut multis in Vergilio locis probatur ", " notons que la concision est le propre des rois, à preuve de nombreux passages chez Virgile.»Cf. Servius auct. ad 10.621: « ubique reges breuiter loquentes ostendit », " partout il montre les rois en train de parler avec concision. »Cf. A. L. Keith, « Briefly speaking in Vergil », dans Classical World 15 (1921/1922), p. 50-51 ; M. Squillante Saccone, 
op. cit., p. 109-110. Ne voir ici que l'application mécanique du conseil «attentos [...] faciemus [...] si pollicebimur nos breui nostram causam demonstraturos", "nous les rendrons attentifs, si nous promettons d'être bref dans la démonstration de notre cause" (Cicéron, De l'invention 1.23) n'apporte rien à l'explication du passage (ainsi Lambert Hortensius ad loc., col. 745 : «attentio a breuitate defensionis ", " attention à partir de la concision de la défense »; ou encore T. E. Page, op. cit., ad loc., p. 370 : "the 'few words' of every orator [...], however lengthy ", "les "peu de mots" de chaque orateur, quelque long soit-il »).

37. Cf. G. Highet, «Speech and narrative in the Aeneid " dans Harvard Studies in Classical Philology 78 (1974), p. 195. Alors qu'Énée hésitait auparavant longuement (v. 284 : «quae prima exordia sumat », « quels mots trouver pour commencer?»), il est ici pris au dépourvu et doit décider en un temps très court comment formuler sa réponse qu'il veut aussi efficace que s'il l'avait longuement préparée.

38. Donat, p. 4,26: « inuenies in poeta rhetorem summum [...] idem enim tibi, ut aliquibus locis exempli causa posuimus, artem dicendi plenissimam demonstrabit ", " tu trouveras sous le poète un très grand rhéteur [...]; en effet, comme nous l'avons signalé pour quelques passages à titre d'exemple, il manifestera un art de la parole accompli »; p. 6,15: «quo fit ut Vergiliani carminis lector rhetoricis praeceptis instrui possit », « il s'ensuit que le lecteur de l'épopée virgilienne peut s’instruire dans les règles de la rhétorique. »

39. En tant que hôtes de Didon, $c f$. R. K. Gibson, « Aeneas as hospes in Vergil, Aeneid 1 and 4 », dans Classical Quaterly 49 (1999), p. 196-198. Dans sa réponse, Didon ne mentionnera plus du tout ce qu'elle appelait leur mariage, mais détaille longuement les services qu'elle a autrefois rendus à ses hôtes (v. 370-378).

40. Ainsi J. C. Scaliger, Poetices libri septem, 3.23 (vol. 2, p. 294,14 Deitz) : «Itaque etiam cum insinuatione ingreditur et appellat: Reginam", "Voilà pourquoi aussi il commence par une insinuation [exorde indirect] et l'appelle Reine »; ou encore J. L. de La Cerda (éd. Cologne, 1628), p. 428 : «Cur Regina ? tum ut colligat beneuolentiam »; mais le savant jésuite ajoute très finement « tum potius ut se subtrahat nuptiis quas Dido obiecerat quae cum Aenea ut cum coniuge loquebatur, sed ille cum illa ut cum regina ", " Pourquoi Reine? soit pour s'attirer la sympathie, soit plutôt pour se dérober au mariage que lui avait opposé Didon qui s'adressait à Énée comme à son époux, alors que lui s'adresse à elle comme à une reine ». Le vocatif Regina se traduit en français le mieux par «Madame» (ce qui exclut évidemment le tutoiement accepté tant par A. Bellessort que J. Perret dans leurs traductions respectives de la C. U. F.).

41. R. G. Austin note ad loc. que Vigile n'utilise jamais une forme oblique de Dido.

42. À noter que l'idée de souvenir (335: «meminisse ») est répétée toute de suite après (336: « dum memor ipse »).

43. Servius ad IV.333 (p. 523,6) : "Controuersia est plena, in qua et purgat obiecta, remouens a se crimen ingrati, et ueniali utitur statu, profectionem suam retorquens in uoluntatem deorum. Habet etiam finem, nam purgat obiectam fugam nomine profectionis. »

44. Selon Servius, l'exorde a déjà une fonction argumentative très précise.

45. Fortunatianus, p. 93,32 Halm, p. 90,4 Calboli Montefusco. Cf. aussi Sulpicius Victor, p. 348,31 Halm : "deprecatio est seu ueniae petitio, cum de eo quod intenditur et confitemur scilicet, ueniam petimus, praetendentes aliquam facti causam quare possit ignosci », « Il y a déprécation ou demande de pardon quand nous demandons pardon pour ce dont on nous accuse et que nous avouons bien sûr, mais en faisant valoir pour cette action un mobile qui rend l'indulgence possible ». Cicéron, Inu. 1.15 parle de « purgatio ». D'où peut-être l'occurrence de « purgare » dans le texte de Donat.

46. Le terme ne doit pas être compris dans l'acception que lui donne la langue juridique actuelle, où il désigne une opération relevant entièrement de l'état de cause de la définition ("quid sit»). Le status generalis ("constitutio iuridicialis» [Rhétorique à Hérennius] ou «quaestio qualitatis» [Quintilien, Institution oratoire]) répond à la question « quale sit », " quelle est la qualification », ou à la question « an iure », « est-ce licite ». 
47. Cicéron, Inu. 1.14-15. Cf. L. Calboli Montefusco, La dottrina degli status nella retorica greca $e$ romana, Hildesheim-Zürich, 1986, p. 129-136.

48. Cf. Cicéron, Inu. 2.94 (trad. G. Achard, Paris, C. U. F. 1994) : «concessio per quam non factum ipsum probatur ab reo, sed ut ignoscatur, id petitur. Cuius partes sunt duae: purgatio et deprecatio. Purgatio est per quam eius qui accusatur non factum ipsum, sed uoluntas defenditur. Ea habet partes tres : imprudentiam, casum, necessitudinem. » «Dans l'aveu l'accusé ne cherche pas à justifier l'acte même qu'il a commis, mais demande qu'on lui pardonne. On y distingue deux parties : l'excuse et la supplication. L'excuse consiste à justifier non pas l'acte même commis par l'accusé, mais les intentions de celui-ci. Il se subdivise en ignorance, hasard malheureux et force majeure. »

49. N. Nascimbeni (éd. Bâle, 1577), col. 742 B : «iuridiciali [sc. quaestione] ex concessione criminis, quod profectionis suae causam in Iouis uoluntatem transfert. »

50. Ph. Melanchthon (éd. Lyon, 1556), p. 213 : « excusatio per translationem. Omnem enim culpam in deos et fata reicit ».

51. J. C. Scaliger, Poetices libri septem, 3.23 (vol.2, p. 294,12 Deitz) : «tota oratio consistebat in dilutione exprobrationis et translatione criminis in fata. Interpretatur enim res ipsas subtilissime; is namque est causae status relatus ad qualitatem ", " tout le discours consiste dans une réfutation des reproches et dans le transfert de l'accusation sur le destin. Il traduit en effet le fond même de manière très subtile ; il s'agit en effet de l'état de cause lié à la qualification ».

52. Ainsi K. Billmayer, op.cit., p.90, qui parle ici de "lusis kat' anatropen " ("réfutation par démolition », en grec). Telle était déjà l'interprétation de M. A. Ferrazzi, Exerc. rhet., p. 27 : « Negat pariter, quod exprobrauerat Dido, et tacitam fugam se cogitasse et quaesiuisse reginae coniugium ", « il nie en bloc ce que Didon lui avait reproché, à la fois d'avoir prémédité sa fuite en cachette et d'avoir cherché à épouser la reine ».

53. Ici encore, Donat évite le terme technique.

54. D'après cette interprétation, «fugam » (au vers 338) doit être attribut de « hanc » (« je n'ai pas espéré cacher ceci comme [si c'était] une fuite »). De même chez Servius, p. 524, 1, qui veut sousentendre "profectionem» (" départ») derrière « hanc ": "nam posteriori non potest iungi, ne sit confessio", "car [l'adjectif démonstratif] ne peut se rattacher au substantif qui suit, sinon il s'agirait d'un aveu. » Il n'est pas moins vrai que cette affirmation du héros est en contradiction avec la description de sa première réaction (281: «ardet abire fuga ») ainsi que des ordres donnés à ses hommes (289-291: "classem aptent taciti [...] et quae rebus sit causa nouandis dissimulent ", « qu'ils appareillent la flotte en silence [...] et qu'ils cachent la raison du revirement »). $C f$. R. Verdière, « Notes de lecture", dans H. Bardon-R. Verdière éd., Vergiliana : recherches sur Virgile, Leiden, 1971, p. 384-385.

55. Donat, p. 403, 19 : «Iunctus sum, inquit, tibi, sed illud non potest coniugium uocari ; non enim semper mulieris ac uiri conuentio matrimonium facit. Aliud uocatur quod gessimus. »

56. Servius auct., p. 524, 5 .

57. Servius auct., p. 524, 20 propose de comprendre « ueni » dans le sens de « consensi »: «non ad haec consensi foedera, ut cum uellem discedere non liceret ", "je n'ai pas consenti à une alliance qui m'interdirait de partir quand je le voudrais ». En fait, Énée souligne ici implicitement que désormais il est de nouveau conscient de sa mission qu'il avait quelque peu négligée et qui ne prévoyait pas qu'il allait débarquer en Afrique pour y demeurer.

58. Aen., 4, 171-172: «nec iam furtiuum Dido meditatur amorem/coniugium uocat, hoc praetexit nomine culpam.» Cf. les remarques moralisantes de Donat, p. 376, 1: «matrimonii nomine turpissimam culpam credidit obtegendam. Verbo iuris usus est ut diceret culpam; sic enim definitum est culpam esse mulieris, cum pudoris in causa peccauerit. Qua autem spe ducebatur Dido matrimonii quod eo genere contraxerat, quae ipsum Aenean in conuiuio referentem audisset responso Creusae uaticinantis accepto et regnum Italiae iam sibi paratum esse et coniugem regiam [...] ? » Nous traduisons : « elle a cru devoir voiler du nom de mariage une culpabilité des plus honteuses. Pour parler de culpabilité, il [sc. le poète] a eu recours à un terme juridique ; en effet, la loi statue qu'une femme 
est coupable dès lors qu'elle a failli dans une affaire de mœurs. Mais comment Didon a-t-elle pu espérer transformer en mariage une relation de ce genre, alors même qu'elle avait entendu, au cours du dîner, Énée expliquer que, d'après la réponse de Créuse l'inspirée, l'attendaient en Italie à la fois un royaume et une épouse royale [...] ? »Cf. aussi V. Marin, «Coniugium uocat, hoc praetexit nomine culpam (Énéide, IV, 172)», dans Les Études Classiques 61 (1993), p. 131-138; J. L. Moles, « Aristotle and Dido's hamartia », dans Greece and Rome 31 (1984), p. 48-54.

59. Donat: " hoc quod facio, inquit, quod nauigare dispono, non est uoluntatis meae nec mihi liberum est uiuere ubi uellem; quod si esset, praestabat remanere me in cineribus patriae, etc. »

60. Cohérent avec l'interprétation qu'il donne du discours d'Énée (« purgatio »), Donat note ad 4.361 (p. 405,23) : « haererent mihi crimina querellis tuis expressa, si erga te non auctoritate multarum metuendarumque iussionum, sed mea uoluntate peccarem ", "les accusations formulées dans tes plaintes m'embarrasseraient si c'était volontairement et non par obéissance à de nombreux ordres redoutables que j'avais commis une faute vis-à-vis de toi.» Or, rien dans le texte ne semble indiquer que Virgile fasse dire à son héros « pecco nolens» («j'ai commis une faute sans le vouloir »). Son départ ne peut être un peccatum ou faute à ses yeux et l'avis de Didon ne mérite pas d'être pris en considération, car elle est « furens » (298) et « impotens animi » (300).

61. Cicéron, Inu. 1.15 : « Ac iuridicialis quidem ipsa in duas tribuitur partes, absolutam et adsumptiuam. Absoluta est quae ipsa in se continet iuris et iniuriae quaestionem ", " [La qualification] judiciaire est subdivisée en deux catégories: l'absolue et l'empruntée. L'absolue contient en elle-même la question du juste et de l'injuste » (trad. d'après G. Achard, C. U. F.). Cf. L. Calboli Montefusco, op. cit., p. 108-113. Selon Quintilien, Inst. 7.4.4, il s'agit là de la ligne de défense la plus efficace : « Defensio longe potissima est qua ipsum factum quod obicitur dicimus honestum esse ", "Le meilleur parti est, de loin, pour la défense de soutenir qu'en lui-même l'acte qui nous est reproché est un acte honorable» (trad. J. Cousin, Paris, C. U. F., 1977).

62. Cf. Hermogène, Sur la constitution des causes, 2.4 (p. 38, 3 Rabe) : « S'il [sc. le débat] concerne un acte à venir, l'état de cause sera pragmatique; en effet l'état de cause pragmatique est une contestation sur un acte à venir : ceci doit-il se faire ou non...? (trad. M. Patillon, Corpus rhetoricum, t. II, Hermogène : les états de cause, C. U. F., 2009). Les Latins parlent de status negotialis. Cf. Sulpicius Victor, p. 342, 2 Halm : "uideamus iam de statibus qualitatis, cuius primus ex omnibus est [...] negotialis. Hic aut legalis est aut rationalis: rationalis cum sine ullo scripto de aequitate rerum disceptatur [...]. Deliberatiuae quoque causae in eundem statum cadunt ", "Voyons à présent les états de la qualification, dont le premier de tous [...] est l'état pragmatique. Ce dernier est soit légal soit logique: logique, quand le débat porte sur l'équité intrinsèque sans référence à une disposition écrite [...]. Les causes délibératives relèvent également du même état de cause ». La notion est assez confuse chez les rhéteurs latins, y compris chez Cicéron (cf. Quintilien, Inst. 3.6.58). Cf. J. O. Ward, "The constitutio negotialis in antique Latin rhetorical theory ", dans Prudentia 1 (1969), n 2, p. 29-48 ; L. Calboli Montefusco, op. cit., p. 99-106.

63. M. Laubanus, Archetyporum analyticorum, p. 100 (tab. XVIII ad librum IV).

64. L'interprétation de cette exclamation par Donat est alambiquée. Il la paraphrase ainsi (p. 404, 10) : «quantum ad humanam pertinet uoluntatem, quod esse potest in potestate mea, amo hanc ciuitatem tanto adfectu ut arbitrer ipsam esse patriam meam », "Dans les limites de la volonté humaine et des moyens en mon pouvoir, je porte à cette cité un amour et une affection tels que j'estime qu'elle est bien ma patrie. » Ce n'est pas spontanément qu'il part pour l'Italie, mais sur ordre des dieux, et il fera tout l'effort humainement possible pour considérer cette nouvelle terre comme sa patrie, pour l'aimer autant que Troie. Seruius (p. 526, 10) explique plus clairement : «eo, inquit, desiderio per uoluntatem deorum iterum circa Italiam teneor quo possem circa Troiam », " Par la volonté des dieux, je suis à nouveau pris pour l'Italie de ce désir dont je serais capable pour Troie. »

65. Au v. 350, fas implique un droit émanant d'une divinité.

66. Nascimbeni a signalé la kakophônia résultant de l'emploi répétée de la syllabe -re- (il aurait pu dire de la quadruple présence de la lettre canine $r$ ) dans cette fin de vers : « quae non fortuito, sed 
de industria facta esse uidetur, ut syllabarum asperitate rei quoque, id est nauigandi et quaerendi extera regna difficultatem significaret", "elle semble être le fruit non du hasard, mais d'un choix délibéré ; le poète veut signaler par la dureté des syllabes la difficulté de l'action, c'est-à-dire de la navigation et de la recherche d'un royaume étranger » (éd. Bâle, 1577, col. 745).

67. J. C. Scaliger, Poetices libri septem, 3.23 (vol. 2, p. 296,9 Deitz) : « uerbis autem inuidioribus animat sententiam », « il s'enflamme et ses propos deviennent plus outrés ». La traduction de Deitz (« mit ziemlich gehässigen Worten ») trahit la pensée de Scaliger qui a qualifié le procédé d'« efficacissimam collationem » (« analogie très efficace »); inuidus doit avoir ici le sens de "outré/révolté » plutôt que de « odieux/révoltant».

68. Le «tandem » du v. 349 exprime l'impatience indignée.

69. J. C. Scaliger, ibid. : "Non quasi hic nos uelis, sed quasi ibi illos nolis », " non pas comme si tu voulais nous [voir] ici, mais comme si tu ne voulais pas les [voir] eux là-bas ».

70. Nascimbeni (éd. Bâle, 1577, col. 745) : « amplificat ex iis quae artis expertia sunt ». Il aurait pu ajouter que la comparatio qui précède est aussi un procédé d'amplificatio (cf. Quintilien, Inst. 8.4.9).

71. Servius, p. 527,3 ad 352: «[Nox operit terras] a uerisimili : ideo tempus posuit », « [la nuit couvre la terre] à partir de la vraisemblance : voilà pourquoi il indiqua le moment »; Donat, p. 405, $1:$ « ut credibile fieret quod dicebat, addidit tempus ", " il ajouta une indication de temps pour que ses propos gagnent en crédibilité ».

72. Servius, p. 528, 1 : « manifesto in lumine aut claro, aut in nimbo cuius maius est lumen » « dans une lumière rayonnante ou éclatante, ou encore dans un nimbe d'une lumière exceptionnelle.» L'épiphanie est un phénomène visuel, l'apparition d'un dieu est toujours rayonnante ( $c f$. Aen. 2.589-590, l'apparition de Vénus à Énée) et cet éclat peut même être dangereux pour un mortel, à en juger par le sort de Sémélé.

73. 357 : "testor utrumque caput ", sans doute les têtes de Jupiter et de Mercure, plutôt que la sienne propre et celle de Didon. L'hésitation remonte à Servius, p. 527,25: « aut meum et tuum, aut Iouis et Mercurii, aut meum et Ascanii » « ou bien la mienne et la tienne, ou celles de Jupiter et de Mercure, ou encore la mienne et celle d'Ascagne». Donat, p. 405,19, a bien tranché : « utrumque caput, hoc est Iouis qui misit et Mercurii qui pertulit », « les deux têtes, celle de Jupiter qui a envoyé et celle de Mercure qui a transmis ».

74. Donat, p. 405,21 : "cognouisti iustas abscessus mei causas, desine me ac te querellis tuis incendere ", «tu connais les raisons légitimes de mon départ, cesse d'attiser de tes plaintes à la fois ton cœur et le mien ». Cf. Nascimbeni (éd. Bâle 1577, col. 745) : «Didonem ne frustra se maceret, ab utili monet ", « à partir du lieu de l'utile, il met Didon en garde de ne pas se miner inutilement. »

75. M. Laubanus, op. cit., distingue deux parties dans cette péroraison : une partie dissuasive (le vers 360 ) et une partie récapitulative. En fait, ce vers 361, inachevé, est aussi la justification de l'ordre intimé à Didon au vers précédent.

76. Cf. J. Pontanus (éd. Augsbourg, 1610), col. 1180 (citation de M. A. Muret) : « intelligendum est aliud esse non sponte facere, aliud inuitum facere. Neque enim quisquis non sponte fecit, inuitus fecisse dicendus este ", « il faut bien comprendre que ne pas agir de sa propre initiative et agir malgré soi font deux. En effet, tout homme qui n'a pas agi de sa propre initiative ne doit pas être considéré comme avoir agi malgré lui ». Cf. aussi J. Henry, Aeneida, vol. 2, Dublin, 1878, p. 710 : «not equivalent to 'inuitus', but equivalent to 'non motu proprio': I am not running after Italy to please myself " (« n'équivaut pas à "contre mon gré”, mais à "pas de mon propre mouvement” : je ne vais pas en Italie pour suivre mon plaisir »). Cf. cependant 6.460-462 (Énée à Didon aux Enfers) : « inuitus, regina, tuo de litore cessi. Sed me iussa deum [...] imperiis egere suis ", " c'est malgré moi, Madame, que j'ai quitté votre rivage. Mais les ordres des dieux [...] m'y ont contraint impérieusement. » Il part peut-être sans plaisir, mais sans se révolter contre son destin. Il est peut-être tiraillé en son for intérieur («inuitus cessi » est en contradiction avec «ardet abire fuga» au vers 281), mais ce tiraillement n'entrave ni même ne retarde son action. 
77. Servius auct., p. 528, 14. Sans rejeter complètement cette interprétation, F. W. Lenz («The incomplete verses in Vergil's Eneid", dans H. Bardon et R. Verdière éd., Vergiliana : recherches sur Virgile, Leiden, 1971, p. 167) envisage sérieusement l'hypothèse que Virgile, au moment de lire le livre IV devant Auguste, ait improvisé un complément tel «sed fata requirunt " ( mais les destins le demandent ») ou "sed numina cogunt» («mais les divinités m'y obligent»), mais que, au moment de la rédaction définitive, il aurait éliminé cet hémistiche improvisé qui risquait d'ôter tout effet à la concision du «non sponte ».

78. Servius auct. ad 362 (362: « Talia dicentem »), p. 528, 15 : «bene "dicentem", non "postquam dixit " ", « il préfère judicieusement "pendant qu'il parle" à "quand il a terminé”. »

79. Donat, p. 4, 26 (ci-dessus n. 38).

80. Saturnales, 5.1.1-2 (trad. d'après F. Richard, Paris, Garnier, 1937) : «omnes inter se consono murmure Vergilium non minus oratorem quam poetam habendum pronuntiabant, in quo et tanta orandi disciplina et tam diligens obseruatio rhetoricae artis ostenderetur. »

\section{AUTEUR}

\section{CLAUDE LOUTSCH}

Université du Luxembourg 